\title{
¿Por qué algunos países sufren exceso de regulación?
}

Víctor Lapuente Giné*

Palabras clave: Carga reguladora, procedimientos administrativos, Economía Politica, elección racional, sistema judicial.

En el lugar de la Mancha donde nombra a Sancho gobernador de la ínsula Barataria, Don Quijote aconseja a éste: «no hagas muchas pragmáticas; y si las hicieres, procura que sean buenas». En Washington, cuatro siglos más tarde, el Banco Mundial llega a conclusiones semejantes: la «carga reguladora», el exceso de regulación o el exceso de procedimientos administrativos tienen un impacto sobre diversos indicadores de desarrollo económico y humano (Informe sobre el Desarrollo Humano 1997). Sin embargo, numerosos indicadores comparados (como los recogidos por Kaufman et al. 1999) constatan que en muchos países existe esa «carga reguladora». Al mismo tiempo, tanto la elaboración, como la aprobación y la posterior implementación de la regulación resultan costosas. Por tanto, existe una paradoja -para la cual no existen explicaciones satisfactorias en la literatura- que se puede formular en los siguientes términos: ¿Por qué hay exceso de regulación en una sociedad si ello tiene unos costes sociales y unos costes para los políticos encargados de elaborar e implementar la regulación? ¿Bajo qué circunstancias optarán los políticos por una decisión aparentemente costosa?

Antes de contestar a estas preguntas es necesario responder a una más básica: ¿qué se entiende en este trabajo por exceso de regulación, exceso de procedimientos administrativos o «carga reguladora»? ¿Acaso no es imprescindible la actividad pública reguladora y unos procedimientos administrativos que aseguren la igualdad de trato para los usuarios de la Administración Pública? Este estudio parte de la premisa de que la regulación no sólo es necesaria para el buen funcionamiento de la actividad económica en una sociedad, sino que, posiblemente, cada día sea un poco más necesaria (Banco Mundial 1997). Sin embargo, también observamos muchos contextos en los que la regulación es más elevada que la cantidad socialmente óptima y ello repercute negativamente en la actividad privada. A pesar de que toda comparación entre distintos contextos entraña dificultades, resulta obvio que hay países con un nivel de regulación más eficiente y países en los que sus ciudadanos sufren una «carga reguladora» o un exceso de regulación ${ }^{1}$. El objetivo de este trabajo no es analizar por qué unos países regulan sobre más materias que otros, ya que la decisión sobre regular un determinado sector obedece a causas muy complejas y distintas para cada país. El objetivo de este estudio es entender por qué, en las materias que regulan, algunos Estados

${ }^{*}$ Nuffield College (Universidad de Oxford) e Instituto Juan March (Madrid).

${ }^{1}$ Para comprobar las diferencias en «carga reguladora» entre diversos Estados dentro de Estados Unidos, véase Scholz (1991). Para observar la fuerte variabilidad existente entre países, véase el trabajo de Kaufman y otros (1999). En el apéndice se presentan los valores de la variable «carga reguladora», extraída del trabajo de Kaufman, para 47 democracias contemporáneas. 
introducen de forma sistemática un exceso de procedimientos administrativos. En este trabajo utilizaré el término carga reguladora (o exceso de regulación) para referirme a dicho exceso de procedimientos administrativos.

El propósito de este artículo es explicar la variabilidad que observamos entre países en el nivel de carga reguladora a través de un modelo teórico que pueda ser generalizable a entornos culturales diversos. La explicación pivota sobre los intereses de los políticos y los dilemas sobre regulación a los que se enfrentan en distintos contextos institucionales. El marco teórico del que parto son las aproximaciones a la regulación basadas en la Economía Política. En concreto, mi trabajo se apoya en los desarrollos teóricos de dos de esas aproximaciones: la Nueva Economía de la Organización (fundamentalmente, Moe 1990; Moe y Caldwell 1994) y la Teoría de la Delegación (en especial, Epstein y O’Halloran 1999; Huber y Shipan 2003). En esos trabajos se reserva a la regulación, entendida tanto como leyes, reglamentos, decretos u otros integrantes del ordenamiento jurídico, el papel de mecanismo utilizado por el principal (el legislativo) para controlar al agente (el ejecutivo). No obstante, las explicaciones del exceso de regulación basadas en la Economía Política presentan numerosos problemas que son analizados en el apartado 2.

Este artículo aporta dos importantes novedades a dicha literatura. A nivel teórico, el modelo descrito en el apartado 3 muestra que, en contra de la hipótesis predominante en la Economía Política, la existencia de un sistema de separación de poderes -o de un sistema con varios 'jugadores con veto'- no produce por sí solo un exceso de regulación. Por el contrario, la presencia de varios jugadores con veto sólo afecta a la carga reguladora en determinadas circunstancias: cuando el sistema judicial es ineficiente y cuando los costes de la regulación no son muy elevados. Tras la formulación del modelo, presento evidencia empírica en la que se muestra cómo la interacción entre el número de jugadores con veto y la eficacia del sistema judicial crea una estructura de incentivos que favorece la elaboración por parte del legislativo de leyes cargadas en exceso de procedimientos administrativos.

\section{Problemas de las explicaciones existentes en la Economía Política sobre la regulación}

La legislación está recibiendo una atención creciente por parte de los científicos políticos. En primer lugar, por su importancia para la teoría de la democracia. Si entendemos la democracia representativa como cadenas sucesivas de delegación (Muller y Strom 2000), las leyes deben verse como un eslabón decisivo, con un papel similar al desempeñado por el voto. A través del voto, los ciudadanos eligen a los políticos. Y a través de las leyes, los políticos eligen el qué y el cómo de la implementación de las distintas políticas públicas. En segundo lugar, porque se observa una notable variabilidad en la extensión y grado de detalle procedimental en las leyes que regulan las mismas materias en distintos contextos político-institucionales. Esta variación no es inocua, sino que produce efectos sobre el desarrollo económico, tal y como el Banco Mundial ha venido subrayando, especialmente desde 1997 (Informe sobre el Desarrollo Humano 1997). Para entender por qué unos determinados contextos producen un mayor exceso de regulación que otros, se han apuntado diversas explicaciones desde la Economía Política. Estas explicaciones presentan numerosas deficiencias, tanto de nivel teórico como empírico, que justifican la elaboración de un modelo explicativo alternativo.

La Economía Política mueve su atención hacia la regulación ante el fracaso de los argumentos que tradicionalmente se han usado en el campo de la administración pública para explicar el exceso de regulación ${ }^{2}$. Una

\footnotetext{
2 Para un resumen crítico de esos argumentos, véase Scholz (1991).
} 
de las explicaciones más recurrentes consiste en achacar la carga reguladora a motivos técnicos. Los legisladores no tienen la preparación técnica necesaria para legislar sobre una materia dada e incluyen más procedimientos administrativos de los que son necesarios para una implementación óptima. Sin embargo, la disparidad entre los niveles de regulación observados en las mismas materias ha llevado a algunos autores a preguntarse sobre la posibilidad de que existan intereses de los políticos detrás. Scholz $(1984,1991)$ se sorprende al ver cómo en ocasiones los beneficiarios de una política contribuyen a incrementar la ineficiencia burocrática en la aplicación de la misma, como sucede en la regulación sobre seguridad en el trabajo en EEUU. En concreto, Scholz comprueba cómo en los Estados con mayoría demócrata en el legislativo se aprueban leyes de regulación en el trabajo cargadas de detalles procedimentales, con unos costes de implementación más elevados y unos resultados en términos de accidentes laborales peores que los Estados con mayoría republicana. La razón para estas observaciones contraintuitivas hay que buscarla en la dinámica del juego político: los legisladores demócratas, influidos por grupos de interés vinculados a los trabajadores, desean atar las manos del ejecutivo (gobernador del Estado) para evitar que éste pueda llegar a acuerdos informales con las empresas reguladas, como, por ejemplo, avisar de antemano cuando se vaya a realizar una inspección a la factoría. Los legisladores republicanos dejan mayor margen de discreción a los gobernadores que, gracias a no tener que seguir unos requisitos procedimentales muy estandarizados, llevan a cabo, en general, políticas más eficientes. Por eficiencia Scholz entiende que se reduzcan tanto los accidentes laborales como los costes burocráticos de implementación del programa, sin que se detecten prácticas fraudulentas de apoyo a determinadas empresas.

Dado que los intereses de los políticos parecen tener un papel destacado a la hora de generar más o menos carga reguladora, diversos autores dentro de la Economía Política -una corriente metodológica que asienta sus explicaciones sobre los intereses de los individuos- han empezado a analizar la regulación. En este apartado, se repasan dos de las contribuciones más destacadas: el trabajo de Moe y Caldwell (1994) y el de Huber y Shipan (2003).

La investigación sobre el exceso de regulación existente en una sociedad no ha podido escapar al debate normativo de presidencialismo versus parlamentarismo. En este sentido, el trabajo de Moe y Caldwell (1994:172) explícitamente considera que «cuando las naciones escogen una forma, presidencialismo o parlamentarismo, están escogiendo un sistema completo, cuyas diversas propiedades surgen endógenamente». Parlamentarismo o presidencialismo son un código genético que se incrusta en todo el sistema, afectando de manera especial al grado de requisitos procedimentales exigibles por y en una administración.

Para Moe y Caldwell, en un sistema presidencial o de separación de poderes, el interés de aquellos encargados de redactar la regulación sería blindar los intereses de sus grupos de apoyo frente a los intereses de los grupos que respaldan al ejecutivo. La preocupación por el grado de eficiencia global de la administración no entraría dentro de las preferencias de los legisladores. Moe y Caldwell (1990: 237) creen que los miembros del legislativo «enterrarán a la burocracia» con leyes cargadas de requisitos y procedimientos formales cuyo objetivo básico es la protección de sus grupos de interés. Por ejemplo, los legisladores pueden introducir en la ley el requisito de que el ejecutivo consulte con determinados grupos de interés antes de realizar una determinada acción implementadora. En esta estructura de separación de poderes, el presidente es el único que se beneficiaría electoralmente de la existencia de una administración pública eficiente. Como respuesta a las imposiciones formales del legislativo, el ejecutivo tratará de crear su propia burocracia autónoma para perseguir cierta eficacia en la aplicación de las normas. Pero el resultado final será un mayor solapamiento entre órganos burocráticos. En los sistemas presidenciales, la Administración pública será, por tanto, «una pesadilla estructural» (Moe 1990:238).

Por el contrario, en un régimen parlamentario, la estructura legal no puede funcionar como mecanismo de protección de los intereses de los grupos de presión que apoyan al legislativo frente a los intereses de los grupos 
que respaldan al ejecutivo. La razón es que ambos intereses son idénticos, dado que los gobiernos en sistemas parlamentarios deben disponer de una mayoría en el legislativo. Expresado en términos más sencillos, no tiene sentido que la mayoría legislativa introduzca unas restricciones procedimentales al funcionamiento de su propio gobierno. Por lo tanto, la predicción de Moe y Caldwell es que no existirá carga reguladora en los sistemas parlamentarios.

La primera crítica que se puede hacer al trabajo de Moe y Caldwell es la escasez de soporte empírico para sus hipótesis. Su evidencia más destacada es la descripción comparada de la acción reguladora sobre emisiones industriales contaminantes en lo que ellos consideran dos casos polares de presidencialismo y parlamentarismo: EEUU y el Reino Unido. Mientras en EEUU la burocracia reguladora actúa de manera ineficiente y cargada de requisitos formales, en el Reino unido, por el contrario, la burocracia encargada de implementar la regulación ambiental tiene más margen para utilizar mecanismos informales y cooperativos con las empresas reguladas. Estos mecanismos informales, sin llevar a la connivencia perversa (aunque Moe y Caldwell tampoco se plantean esta hipótesis seriamente), conducen a unos resultados más eficientes: menos costes de implementación y más control de emisiones contaminantes. El primer problema con esta evidencia es que Moe y Caldwell no definen la variable latente en cuyos extremos supuestamente se encuentran el Reino Unido y EEUU. No sabemos por qué el Reino Unido es, por ejemplo, más polar que otros sistemas parlamentarios como Portugal, Italia o España. El segundo problema es que no está claro que la regulación ambiental estudiada por Moe y Caldwell sea significativa del grado de carga reguladora existente en una sociedad. Como se puede ver en el apéndice -en el que se recoge el indicador regulatory burden (carga reguladora) creado por Kaufman para el Banco Mundial- el valor para el Reino Unido $(1,206)$ es muy similar al valor para EEUU $(1,135)^{3}$. De forma que, el caso «polar» de parlamentarismo y el caso polar de presidencialismo tienen menos carga reguladora que otros casos «no polares» de parlamentarismo (como Italia o Israel) o de presidencialismo (como Argentina o Brasil). Mi intención en este trabajo es aportar evidencia que vaya más allá de estos dos casos polares. De esta forma, en el apartado 4 se realiza un análisis cuantitativo con una muestra de 47 democracias contemporáneas.

Una segunda crítica que se puede hacer al trabajo de Moe y Caldwell es sobre su planteamiento teórico. En su conocido artículo de 1995, Tsebelis formalizó el argumento de que la línea divisoria en los regímenes políticos democráticos no debe establecerse entre presidencialismo y parlamentarismo, sino entre uno y múltiples jugadores con veto. La definición de jugador con veto sería la siguiente: aquel actor individual o colectivo cuya concurrencia es necesaria para modificar el statu quo. Hay sistemas parlamentarios con un solo jugador con veto (cuando el partido en el gobierno tiene mayoría parlamentaria) y sistemas parlamentarios con múltiples jugadores con veto (cuando existe un gobierno de coalición o cuando el partido en el gobierno necesita apoyos legislativos de otros partidos). A su vez, existen sistemas presidenciales con un solo jugador con veto (el mismo partido controla la presidencia y las cámaras legislativas) y sistemas presidenciales con múltiples jugadores con veto (un partido controla la presidencia y otro u otros controlan el legislativo).

Traduciendo la idea de Tsebelis a los parámetros de nuestra discusión, un sistema parlamentario como el italiano, con varios jugadores con veto, debería tener un nivel de carga reguladora más semejante al de EEUU, que suele tener varios jugadores con veto, que al del Reino Unido, en el que el partido en el gobierno dispone de una sólida mayoría parlamentaria. En este trabajo optaré por el concepto de jugadores con veto porque creo que recoge mejor que la división presidencialismo-parlamentarismo el argumento que Moe y Caldwell tienen en

${ }^{3}$ Carga reguladora es una variable estandarizada de media 0 y desviación típica 1; cuanto más bajo sea el valor, mayor es el grado de exceso de regulación existente en un país. Por ejemplo, Nueva Zelanda presenta uno de los niveles de regulación más óptimos, con un valor de 1,204 y la India uno de los peores de las democracias estudiadas, con -0,040. 
mente al establecer la diferencia entre distintas formas de regular. Para estos autores, el régimen de separación de poderes produce un exceso de carga reguladora cuando existe la posibilidad de que los redactores de la ley puedan encapsular los intereses de sus grupos de apoyo, atando las manos tanto del ejecutivo como de futuros legislativos. Pero si el ejecutivo en un sistema presidencialista pertenece al mismo partido que la mayoría legislativa, ésta pierde cualquier tipo de incentivo para hacer una regulación cargada de procedimientos administrativos. En este caso, que se catalogaría como un único jugador con veto, estaríamos de facto ante un sistema de incentivos idéntico al de un régimen parlamentario. Por tanto, la diferencia relevante respecto al tamaño y detalle de la legislación debe ser entre uno y múltiples jugadores con veto.

Otra explicación interesante sobre el tipo de regulación es la ofrecida por Huber y Shipan (2003). En su intento de construir una teoría comparativa de la delegación, descubren que no existe un patrón propio de cada materia regulada o de la cultura específica de cada país que explique la longitud y el grado de detalle de las leyes. Así, el país X puede tener una legislación sobre control de emisiones contaminantes más extensa que la del país Y; pero, a su vez, el país Y puede disponer de una legislación laboral más extensa que el país X. Enfrentados con el mismo asunto, legisladores en distintas situaciones (más o menos conflicto con el ejecutivo, por ejemplo) parecen tomar diferentes opciones respecto al diseño de las leyes. Los legisladores, si son conscientes de que el ejecutivo implementador de la política tiene unas preferencias muy distintas de las del legislador mediano, incluirán una mayor carga reguladora a la hora de redactar la ley.

Huber y Shipan entienden que la «longitud» de la legislación -entendida literalmente como número de palabras que contienen las leyes- es una buena aproximación del grado de carga reguladora que el legislativo impone al ejecutivo. Además, la longitud de la ley «viaja» bien, ya que en todos los países hay que hacer lo mismo: contar el número de palabras de las leyes. Sin embargo, se puede argumentar que el indicador utilizado por Huber y Shipan está sesgado al centrarse de forma exclusiva en el papel de una ley concreta. El nivel de discreción que el legislativo concede al ejecutivo en, por ejemplo, legislación laboral -que es la legislación analizada por Huber y Shipan en su estudio comparativo- depende de las leyes específicas sobre relaciones laborales, pero también del resto de leyes sobre otras materias que incluyan aspectos laborales. Así, leyes sobre igualdad sexual o sobre salud pública pueden regular aspectos de las relaciones laborales no cubiertos por la legislación específicamente laboral. De hecho, la interdependencia entre políticas públicas, el solapamiento de regulaciones sobre un mismo asunto desde varias legislaciones específicas, es un fenómeno creciente en opinión de numerosos estudiosos de políticas públicas (Subirats 1989). Por otro lado, el legislativo no tiene incentivos para introducir carga reguladora en las leyes ordinarias si ya existe una Ley de Procedimiento Administrativo lo suficientemente limitadora de la discreción de la burocracia al servicio del ejecutivo. Existe, por tanto, todo un ordenamiento jurídico, más allá de la concreta ley, que también puede actuar como freno a la discreción del ejecutivo y que puede suponer una carga reguladora para la sociedad.

La pregunta es ¿podemos encontrar una aproximación (o variable proxy) del nivel de carga reguladora que soporta una sociedad que funcione mejor que la medición de la extensión de leyes concretas y que, además, «viaje» bien de un país a otro? La respuesta que ofrezco en este trabajo -la variable compuesta regulatory burden (carga reguladora) diseñada por Kaufman, Kray y Zoido-Lobatón (1999) para el Banco Mundial (para detalles sobre su composición ver apartado 5) - no está exenta de problemas, porque se trata de una recolección de percepciones subjetivas de los usuarios de las administraciones públicas. Asumir que esas opiniones recogen el nivel real de carga reguladora de cada país resulta complicado. Sin embargo, a mi juicio, no existe mejor alternativa que estas opiniones, porque todavía no se ha creado ningún indicador comparado capaz de medir con precisión el nivel de papeleo o carga reguladora. Además, como señalan los autores del indicador carga reguladora, una de las mejores pruebas de la fiabilidad de las opiniones subjetivas es que hay empresas dispuestas a pagar para obtener 
esa información. Si se trata de una inversión útil para ellas, es porque estos indicadores de alguna forma están reflejando la calidad de la regulación en los distintos Estados (Kaufman et al. 1999).

Otra objeción al uso de esta variable es que no hace distinción entre la regulación de la administración pública -el nivel de procedimientos administrativos, lo que hemos definido como carga reguladora- y la extensión de la regulación pública a sectores sociales o económicos. Se trata de problemas distintos y el objeto de este artículo es entender por qué, a la hora de regular un determinado sector social o económico, los legisladores deciden introducir una carga reguladora o un exceso de procedimientos administrativos. Queda fuera del alcance de este trabajo explicar por qué los legisladores deciden regular un mayor o menor número de sectores. Sin embargo, se puede argumentar que los ciudadanos perciben de forma conjunta los efectos de los dos problemas. Por lo tanto, en el contraste empírico desarrollado en el apartado 4 se hace necesario introducir una variable para controlar hasta qué punto la percepción de los ciudadanos no obedece a que entiendan que el Estado regula muchos sectores, sino que obedece a que los ciudadanos consideran que el Estado impone demasiados procedimientos administrativos en los sectores que regula. Como se verá en el análisis empírico, dicha variable no aparece como significativa, con lo que podemos afirmar que nuestra variable dependiente -carga reguladora- es una buena aproximación del nivel de procedimientos que el Estado introduce en las materias que regula. Podemos comprobar este punto si observamos el indicador que aparece en el apéndice, ya que países con una fuerte tradición de regulación social y económica, como son los países escandinavos, presentan, sin embargo, unos índices de carga reguladora percibida muy baja. Por tanto, parece que los ciudadanos son capaces de distinguir ambos fenómenos.

\section{Una propuesta de modelo teórico para explicar la carga reguladora}

Siguiendo las aproximaciones de la Economía Política al problema de la regulación, el modelo que se describe a continuación también parte de los intereses de los políticos. Como señala North (1990:16), las leyes, como el resto de instituciones, «son creadas para servir los intereses de aquellos con el poder negociador para imponerlas». North insiste en los costes de negociación, o de forma más general en los costes de transacción, como clave para entender porqué en ocasiones los políticos prefieren instituciones ineficientes. Aplicado a nuestro caso, los políticos, al negociar con otros políticos de preferencias distintas, enfrentan unos costes de negociación que pueden derivar en la adopción de leyes que impongan una carga reguladora a la sociedad.

El supuesto del que parto, compartido en general por la literatura, es que los políticos están interesados en su reelección. Para simplificar, utilizo sólo dos actores: el legislativo, que representa la posición del legislador mediano, y el ejecutivo, que representa al gobierno. El mecanismo principal que tienen ambos actores para conseguir su reelección es elaborar una regulación que sea percibida como óptima por los votantes. Es decir, que no esté cargada de procedimientos administrativos que lastren el desarrollo económico de la sociedad. Sin embargo, el ejecutivo, por su condición de implementador de la legislación ${ }^{4}$ dispone también de un mecanismo secundario para obtener la reelección: el uso de prácticas corruptas. Es decir, proporcionar favores políticos a empresas o

${ }^{4}$ El ejecutivo utiliza para la implementación la burocracia pública a su servicio. Siguiendo a Huber y Shipan (2003), asumo que el Ejecutivo es un «actor privilegiado» sobre la burocracia, de tal forma que sus preferencias se convierten en las preferencias de la burocracia. La burocracia es un mero instrumento del ejecutivo. Se trata de una asunción que no es realista y, posiblemente, un modelo más completo debería tener en cuenta las diferentes preferencias entre el ejecutivo y la burocracia. Sin embargo, ante la dificultad de testar esas diferencias en un estudio comparativo, opto aquí por la simplificación de un modelo que me permite generar proposiciones que sí son testables. 
particulares a cambio de financiación u otro tipo de apoyo para que el político obtenga la reelección (como respaldo en los medios de comunicación, o ayuda para la movilización electoral). No obstante, el uso de este mecanismo secundario no está exento de riesgos. El sistema judicial, que se asume independiente tanto del legislativo como del ejecutivo, puede detectar la práctica corrupta y sancionar al político. Para analizar la decisión del ejecutivo sobre este mecanismo secundario, utilizo el siguiente esquema de comparación de utilidades esperadas 5 :

$$
(1-p) b_{1}+p\left(-b_{1}\right)>0
$$

donde $b_{1}$ es el beneficio en la carrera electoral que obtiene el ejecutivo procedente de las empresas o particulares a los que ha otorgado favores políticos ilegales, como, por ejemplo, dinero u otro tipo de ayuda para la movilización de los electores a su favor. p mide la probabilidad de que el sistema judicial detecte la práctica corrupta. $-\mathrm{b}_{1}$ es el castigo que el político recibe si la práctica corrupta es sancionada por los tribunales. Si el beneficio de la corrupción es electoral, la penalización también es electoral: b1 entra con signo negativo porque la corrupción gubernamental supone un beneficio electoral para el partido en la oposición. Para simplificar el modelo se supone que el beneficio y el perjuicio son idénticos. En caso de no entrar en prácticas corruptas, el Ejecutivo obtiene el pago 0 en este mecanismo secundario. Es fácil comprobar que la probabilidad p de corte que hace indiferente al ejecutivo entre hacer uso o no de la corrupción es $1 / 2$. Con un sistema judicial que actúe con una probabilidad superior a 1/2, el político, que asumimos neutral al riesgo, tendrá una utilidad esperada negativa en la «lotería de la corrupción», y actuará dentro de los márgenes de la ley. Por el contrario, para probabilidades de actuación de los jueces menores que $1 / 2$, el ejecutivo preferirá usar las prácticas corruptas.

\subsection{Cuando existen varios jugadores con veto}

Analizo a continuación el caso en el que existen dos jugadores con veto (el legislativo está en manos de un partido y el ejecutivo en manos de otro), para estudiar posteriormente el caso en el que sólo hay un jugador con veto (tanto legislativo como ejecutivo pertenecen al mismo partido). Para simplificar el modelo, se asume que legislativo y ejecutivo son actores homogéneos, sin jugadores con veto internos. El modelo podría completarse con juegos entre cámaras legislativas o entre miembros de una coalición en el gobierno, así como introduciendo la burocracia como un jugador con veto ${ }^{6}$. Pero sería necesario analizar antes si el sacrificio de parsimonia al introducir más actores es compensado por hallazgos sustantivos lo suficientemente importantes. En mi opinión, creo que incluir más actores en el modelo añadiría una complejidad técnica al mismo que sólo haría menos inteligibles los resultados y que no aportaría conclusiones relevantes.

${ }^{5}$ Bendor et al. (2001), en su revisión de modelos formales aplicados a la delegación, utilizan una notación similar. El político optará por corromperse aceptando un soborno si, asumiendo neutralidad al riesgo, la cantidad esperada de la lotería (1-p)b -pc es mayor que 0; siendo p la probabilidad de ser detectado por el sistema judicial, c el castigo en caso de ser detectado y b el pago obtenido por el soborno.

${ }^{6}$ El propio Tsebelis $(1995,2002)$ considera que, en determinadas ocasiones, además de a los partidos, es necesario considerar a la burocracia como un veto player. Sin embargo, en este caso no resultaría posible testar las predicciones de un modelo en el que incluyéramos una burocracia con preferencias distintas a los políticos dado que no existen indicadores comparados al respecto. En cambio, sí existen índices comparados que midan cuándo hay discrepancia entre las preferencias del legislativo y el ejecutivo. 


\section{CUADRO 1 \\ El dilema de la regulación con dos jugadores con veto}

\begin{tabular}{|c|c|c|}
\hline $\begin{array}{ll}\text { EJE } \\
\text { LEG }\end{array}$ & Cumplir & Defraudar \\
\hline Regulación Óptima & $\mathrm{x} \quad ; \mathrm{y}$ & $x-\left[(1-p) b_{1}+p\left(-b_{1}\right)\right] \quad ; \quad y+\left[(1-p) b_{1}+p\left(-b_{1}\right)\right]$ \\
\hline Regulación Excesiva & $\mathrm{x}-\mathrm{r} ; \mathrm{y}-\mathrm{r}$ & $\mathrm{x}-\mathrm{r}-\left[(1-\mathrm{p}) \mathrm{b}_{2}+\mathrm{p}\left(-\mathrm{b}_{2}\right)\right] \quad ; \quad \mathrm{y}-\mathrm{r}+\left[(1-\mathrm{p}) \mathrm{b}_{2}+\mathrm{p}\left(-\mathrm{b}_{2}\right)\right]$ \\
\hline
\end{tabular}

Para facilitar comparaciones de utilidad, la estructura de pagos se basa en probabilidades de reelección. El legislativo parte de una probabilidad de reelección x y el ejecutivo de una probabilidad y. Ambas probabilidades están determinadas por factores exógenos al modelo, como la identidad de partido o los rendimientos de gobierno. El legislativo y el ejecutivo mantienen un juego interactivo con dos cursos de acción disponibles para cada uno. El legislativo puede elaborar una regulación óptima, es decir, una ley óptima socialmente en el sentido de Scholz (1991): con los requisitos procedimentales que minimicen los costes de implementación y maximicen los resultados de la política. $\mathrm{O}$, por el contrario, puede crear una regulación excesiva, lo que hemos definido anteriormente como carga reguladora, y que consistiría en la introducción de numerosos procedimientos administrativos. La regulación excesiva lleva a un aumento en los costes de elaboración e implementación (representados por la letra $\mathrm{r}$ para los dos actores) y a unos resultados finales ineficientes. Por tanto, disminuye el potencial electoral de los dos actores: obliga al legislativo a dedicar un mayor tiempo y esfuerzo a la elaboración de la ley y a no que regular otras materias de interés ${ }^{7}$. Y obliga al ejecutivo a un mayor esfuerzo en la implementación, así como también incrementa el descontento de los usuarios de la Administración, con lo que el efecto será una reducción en su probabilidad de reelección. Para simplificar el modelo se asume que, en ambos casos, el coste del exceso de regulación entra restando en los pagos y tiene el valor $\mathrm{r}$.

El ejecutivo, por su parte, puede cumplir la ley o puede defraudar, cayendo en prácticas corruptas con empresas o particulares afectados por la regulación y haciendo uso, por tanto, de lo que he denominado mecanismo secundario para obtener la reelección. La lotería de la corrupción $[(1-\mathrm{p}) \mathrm{b}+\mathrm{p}(-\mathrm{b})]$ entrará sumando en los pagos del ejecutivo y restando en los del legislativo, pues el beneficio de la corrupción para uno, como he señalado anteriormente, es el perjuicio para el otro y viceversa. Ahora bien, los potenciales beneficios/perjuicios de la corrupción son distintos en función del tipo de regulación. Un regulación óptima deja más margen de discreción para el ejecutivo, que lo puede utilizar para cumplir eficazmente la ley o bien, para llevar a cabo prácticas corruptas. Sin embargo, si la ley es más detallada y exige más procedimientos que los óptimos, el margen de discreción del ejecutivo es más limitado, recortándose también el margen de corrupción disponible. Traducido a términos del modelo, el beneficio/perjuicio de la corrupción con regulación óptima $\left(\mathrm{b}_{1}\right)$ es mayor que el beneficio/perjuicio de la corrupción con regulación excesiva $\left(\mathrm{b}_{2}\right)$.

Las combinaciones entre las distintas estrategias dan lugar al espacio matricial del cuadro 1. Para la sociedad, el resultado ideal sería el representado por la casilla superior izquierda: un nivel de regulación óptimo y cum-

${ }^{7}$ Huber y Shipan (2003) consideran que los costes de la regulación son muy importantes y, sin embargo, normalmente quedan fuera de los modelos teóricos. Para ellos, reducir la discrecionalidad del ejecutivo, introduciendo detalles en la ley, ya sean estos procedimentales o sustantivos, tiene tanto unos costes directos (tiempo y esfuerzo invertidos en la redacción) como unos costes de oportunidad (no redactar otras leyes). 
plimiento por parte del ejecutivo. Porque, en ese caso, la sociedad no pagaría ni los costes derivados de la regulación excesiva ni los costes de la existencia de prácticas corruptas que benefician a unos intereses concretos. Pero, dadas las interacciones entre los intereses políticos, las condiciones para llegar a ese óptimo social se complican. A continuación, presento los distintos posibles equilibrios agrupados en dos grandes casos: cuando p $>1 / 2$ y, por tanto, la estrategia dominante para el ejecutivo es Cumplir, y cuando $\mathrm{p}<1 / 2$, entonces el ejecutivo sigue la estrategia dominante de Defraudar.

\section{Caso 1: Sistema Judicial Eficiente $(p>1 / 2)$}

Como la lotería de corrupción tiene un valor esperado negativo, la columna cumplir domina fuertemente a la columna defraudar para el ejecutivo: $y+\left[(1-p) b_{1}+p\left(-b_{1}\right)\right]<y$, en el caso de regulación óptima; $y-r+[(1-p)$ $\left.\mathrm{b}_{2}+\mathrm{p}\left(-\mathrm{b}_{2}\right)\right]<\mathrm{y}-\mathrm{r}$, en el caso de regulación excesiva. El legislativo, sabiendo que se va a cumplir la ley, preferirá una regulación óptima, alcanzándose así el socialmente deseable cuadrante superior izquierdo, con pagos x,y.

\section{Caso 2: Sistema Judicial Ineficiente $(p<1 / 2)$.}

En este caso, la lotería de corrupción tiene un valor esperado positivo para el ejecutivo y negativo para el legislativo. La columna defraudar domina fuertemente a la columna cumplir: y $+[(1-p) b 1+p(-b 1)]>y$, en el caso de regulación óptima; $y-r+[(1-p) b 2+p(-b 2)]>$ y -r, en el caso de regulación excesiva. Consciente de la opción por defraudar que tomará el ejecutivo, el legislativo elegirá regular en exceso si:

Utilidad esperada de regulación excesiva > Utilidad esperada de regulación óptima

$$
x-r-\left[(1-p) b_{2}+p\left(-b_{2}\right)\right]>x-\left[(1-p) b_{1}+p\left(-b_{1}\right)\right]
$$

Si despejamos $r$, nos encontramos que el legislativo optará por una regulación excesiva cuando $\mathrm{r}<(1-$ $2 \mathrm{p})\left(\mathrm{b}_{1}-\mathrm{b}_{2}\right)$. Por tanto, si los costes de la regulación ( $\mathrm{r}$ ) son lo suficientemente pequeños, el legislativo puede optar por introducir muchos requisitos procedimentales en la ley. En función del valor de corte $p=1 / 2$, se puede definir el espacio de equilibrios representado en la figura 2, con los posibles valores de p (sistema judicial) en el eje horizontal y con los valores de $\mathrm{r}$ (costes de la regulación excesiva) en el vertical.

\section{CUADRO 2}

Espacio de equilibrios para dos jugadores con veto

\begin{tabular}{|c|c|c|}
\hline $\begin{array}{c}\text { Costes altos de regulación } \\
\mathrm{r}>\left(\mathrm{b}_{1}-\mathrm{b}_{2}\right)(1-2 \mathrm{p})\end{array}$ & $\begin{array}{c}\text { Regulación Óptima } \\
\text { Defraudar }\end{array}$ & $\begin{array}{c}\text { Regulación Óptima } \\
\text { Cumplir }\end{array}$ \\
\hline $\begin{array}{c}\text { Costes bajos de regulación } \\
\mathrm{r}<\left(\mathrm{b}_{1}-\mathrm{b}_{2}\right)(1-2 \mathrm{p})\end{array}$ & $\begin{array}{c}\text { Regulación Excesiva } \\
\text { Defraudar }\end{array}$ & \\
\hline & $\begin{array}{c}\text { Sistema judicial ineficiente } \\
\mathrm{p}<1 / 2\end{array}$ & $\begin{array}{c}\text { Sistema judicial eficiente } \\
\mathrm{p}>1 / 2\end{array}$ \\
\hline
\end{tabular}




\subsection{Cuando sólo existe un jugador con veto}

En el caso de que tanto el ejecutivo como el legislativo estén en manos del mismo partido, la interacción se simplifica considerablemente. La lotería de la corrupción sólo entra en el pago del ejecutivo. Podría introducirse también en el pago del legislativo, aunque en este caso, como el ejecutivo y el legislativo pertenecen al mismo partido, la lotería de la corrupción entraría sumando en lugar de restando. Sin embargo, los resultados sustantivos no cambiarían y, para simplificar, la lotería de la corrupción aparece solamente en los pagos del implementador. Como vemos en la figura 3, para el legislativo la fila regulación óptima domina fuertemente a la fila regulación excesiva: $\mathrm{x}>\mathrm{x}$-r (para ambos casos). Por tanto, el legislativo siempre escogerá regulación óptima. El equilibrio final que se alcance (regulación óptima/cumplir o bien regulación óptima/defraudar) dependerá en exclusiva de la utilidad esperada de la lotería de corrupción. Si el sistema judicial es eficiente ( $p>1 / 2)$, el equilibrio será regulación óptima/cumplir, mientras que si el sistema judicial es ineficiente $(\mathrm{p}<1 / 2)$, se alcanzará la casilla regulación óptima/defraudar.

CUADRO 3

El dilema de la regulación para un jugador con veto

\begin{tabular}{|l|l|lc|}
\hline \multicolumn{1}{|c|}{ EJE } & \multicolumn{1}{c|}{ Cumplir } & \multicolumn{2}{c|}{ Defraudar } \\
\hline LEG & \multicolumn{1}{c|}{$; y+\left[(1-p) b_{1}+p\left(-b_{1}\right)\right]$} \\
\hline Regulación Optima & $x ; y$ & $x$ & $; y-r+\left[(1-p) b_{2}+p\left(-b_{2}\right)\right]$ \\
\hline Regulación Excesiva & $x-r ; y-r$ & $x-r$ & $x-y$ \\
\hline
\end{tabular}

En resumen, el modelo teórico muestra que el hecho de que haya un único o múltiples jugadores con veto no afecta por sí mismo a la decisión del legislativo de optar entre un tipo u otro de regulación. Depende del valor de otros parámetros. Sólo cuando la capacidad del sistema judicial está por debajo de un determinado umbral, y siempre y cuando los costes de elaborar una sobrerregulación no sean muy elevados, el legislativo en la situación de dos jugadores con veto estará dispuesto a cambiar su estrategia natural de elaborar una regulación óptima. Si el sistema judicial está por encima de ese umbral, el efecto perverso que el número de jugadores con veto tiene sobre el exceso de regulación desaparece. Por tanto, el resultado sustantivo que el modelo añade a la hipótesis compartida mayoritariamente en la literatura -sobre la influencia de los jugadores con veto en el tamaño de la legislación- es que esa influencia depende de la interacción con otros factores, en concreto el sistema judicial y los costes de la regulación. En el análisis empírico que se detalla a continuación, me limito a realizar un contraste de la interacción entre los jugadores con veto y el sistema judicial. Los costes de la regulación quedan fuera del estudio, porque no es posible encontrar indicadores comparados sobre los mismos. La asunción, por tanto, del análisis empírico es que los costes de la regulación son bajos. Se trata de una premisa plausible dado que estamos estudiando Estados, cuyos legisladores suelen disponer de los medios suficientes para redactar leyes cargadas con procedimientos. En el caso de que estudiáramos regiones u otros ámbitos subnacionales, esta asunción sería más problemática.

\section{Contraste empírico}

\subsection{Variable dependiente: carga reguladora}

La variable a explicar es la carga reguladora (regulatory burden), creada por Kaufman et al (1999) para el World Bank Institute for Governance, Regulation and Finance. La variable es un índice compuesto de indicadores recogidos 
entre los años 1997 y 1999 y procedentes de dos fuentes. Por un lado, sondeos a expertos, que reflejan los índices de cada país producidos por agencias de «valoración de riesgo» y otras organizaciones. Y, por otro lado, encuestas a residentes de cada país llevadas a cabo por organizaciones internacionales y organizaciones no-gubernamentales. Los indicadores que conforman el índice compuesto carga reguladora miden desde el nivel percibido de «red tape» (papeleo) al «exceso de regulación para iniciar negocios» o los «impedimentos a la exportación». El denominador común de todos ellos sería la percepción de que existe una carga reguladora: los ciudadanos deben lidiar con excesivos procedimientos administrativos. Como se ha mencionado con anterioridad, carga reguladora es una variable estandarizada de media 0 y desviación típica 1. Cuanto mayor sea el valor, menor es el grado de carga reguladora existente en un país. Por ejemplo, el Reino Unido $(1,206)$ tiene mucha menor carga reguladora que Venezuela $(0,090)$.

Dado que el modelo teórico desarrollado en este trabajo parte de que la legislación es fruto de mecanismos democráticos, quedan fuera del análisis empírico los regímenes autocráticos. De esta forma, la muestra de países seleccionados está compuesta por las democracias de todo el mundo en el periodo 1981-1995 con población superior al millón de habitantes. Los datos sobre democracia se han extraído de la base de datos de Álvarez, Cheibub, Limongi y Przeworski (1997). Para no excluir algunos casos relevantes (por ejemplo, Argentina), he incluido también aquellos países en los que el número de años de dictadura dentro de ese periodo no excediera los cuatro.

\subsection{Variables independientes}

La variable jugadores con veto procede del Data Base Political Institutions del Banco Mundial. Se trata de una variable que tiene en cuenta tanto lo que Tsbelis (1995) denomina jugadores con veto «institucionales» (si hay bicameralismo o no, si el presidente es elegido directamente o no) como los jugadores con veto «partidistas» (si el partido del presidente es el mismo que el que controla las cámaras o no). El número final para cada país es la media del número de jugadores con veto en el periodo 1981-1995. El intervalo de tiempo es lo suficientemente amplio como para esperar que, en caso de existir alguna influencia del número de los jugadores con veto sobre el nivel de regulación, quede reflejada en el análisis estadístico.

La eficiencia del sistema judicial es una variable difícil de medir y sobre la que no existen indicadores objetivos que permitan realizar estudios comparativos. De nuevo, creo que la mejor aproximación para conocer la eficiencia del sistema judicial son las percepciones subjetivas de expertos y usuarios del sistema judicial de cada país. Por ello, he seleccionado otra de las variables compuestas del trabajo de Kaufman et al. (1999): la variable rule of law, creada sobre la base de diversos indicadores de organizaciones internacionales que tratan de medir hasta qué punto los agentes tienen confianza en el sistema legal de cada país. Fundamentalmente, tratan de recoger si el sistema judicial es percibido como eficiente y previsible.

En el análisis empírico se incluyen también tres variables de control. La primera es el PIB per capita, que permitirá comprobar si el nivel de carga reguladora obedece a las variables político-institucionales del modelo teórico o más bien es sólo una consecuencia del desarrollo económico de cada país. En concreto, he introducido el PIB per capita en dólares internacionales para el año 1989 (año intermedio del periodo 1981-1995) para cada una de las democracias estudiadas, con datos extraídos del World Development Report del Banco Mundial.

En segundo lugar, como se ha adelantado en el apartado 2, es posible que la carga reguladora observada por los usuarios de la Administración sea debida a que estamos frente a un Estado intervencionista que regula muchos aspectos de la actividad social y económica del país y no al hecho de que exista un exceso de procedi- 
mientos administrativos. Para controlar ese efecto, incluyo una aproximación de tipo cuantitativo sobre el grado de intervensionismo estatal: el porcentaje de impuestos sobre el total del PIB, con datos extraídos del World Development Report del Banco Mundial también para el año 1989. En contra del uso de esta variable se puede argumentar que las regulaciones generan poco gasto público y que son las políticas distributivas las que lo crean. Sin embargo, y a pesar de que no existen estudios cuantitativos al respecto, podemos ver cómo los Estados que regulan muchos sectores sociales y económicos - por ejemplo, Suecia o Alemania- poseen grandes sectores públicos e imponen un elevado porcentaje de impuestos sobre el total del PIB. Por tanto, aunque no recoja con totalidad el grado de intervencionismo estatal en la sociedad, la variable porcentaje de impuestos sobre el total del PIB es una buena aproximación al mismo.

En tercer lugar, es necesario incluir alguna variable que haga referencia a las distintas tradiciones jurídicoadministrativas de cada país. Con ese objeto se ha introducido una variable dicotómica con valor 1 para los países de tradición Common Law y valor 0 para los de tradición Civil Law, con datos obtenidos a partir del trabajo de La Porta et al. (1999). A pesar de que, obviamente, un único indicador no puede recoger todos los matices de las complejas tradiciones jurídicas o administrativas de cada país, existen varios motivos por los cuales es relevante su inserción en este estudio. En primer lugar, como señalan Huber y Shipan (2003), en los países de Common Law, los legisladores tienen más incentivos para introducir detalles procedimentales en las leyes. La razón es que los jueces del Common Law, por su origen normalmente ajeno a la estructura funcionarial del Estado, no son tan aliados del Estado, como los jueces del Civil Law. Por ello, los legisladores deben ser muy precisos en sus leyes para evitar interpretaciones de la ley que sean hostiles a los intereses de los legisladores y favorables a los intereses de particulares o del Ejecutivo. En segundo lugar, los países de Civil Law suelen disponer de un Derecho Administrativo para las relaciones entre personas jurídicas y el Estado que está separado del Derecho común. Esta separación puede tener efectos contradictorios sobre la carga reguladora de un país. Por un lado, esto podría conducir a una mayor regulación legislativa en los países del Common Law, porque los legisladores no cuentan con unos tribunales de lo contencioso-administrativo que velen por sus intereses. Pero, por otro lado, también es cierto que la existencia del derecho administrativo separado del Derecho común (propia de los países del Civil Law) puede ser percibida como un aumento de la carga reguladora, pues normalmente el Derecho Administrativo obliga a que, en las relaciones con la Administración, deban seguirse unos largos procedimientos y que, sólo tras agotarse éstos, el particular pueda dirigirse a la justicia ordinaria. En conclusión, existen argumentos teóricos que justificarían una influencia del Common Law sobre el nivel de carga reguladora, aunque la influencia podría ser tanto de signo positivo como negativo.

Por último, la existencia de federalismo es vista por la teoría de la delegación como relevante para explicar el nivel de discreción otorgado por un principal (legislador) hacia el agente (ejecutivo federal o regional). La predicción teórica es que, en presencia de federalismo, el legislador introducirá más detalles procedimentales en la ley, puesto que, al menos en parte, la implementación de la política puede quedar en manos de «otro» ejecutivo que no es el suyo propio, sino el de algún ámbito subnacional (Epstein y O’Halloran 1999). Sin embargo, no introduzco dicha variable en el análisis -tras comprobar previamente que no tiene efecto sobre la variable dependiente. El motivo es que el federalismo está altamente correlacionado con el número de jugadores con veto, ya que, por ejemplo, los países federalistas suelen disponer de legislativos bicamerales, que aumentan el número de jugadores con veto del sistema. Por tanto, el efecto del federalismo queda parcialmente recogido en la variable jugadores con veto.

\subsection{Resultados}

Los principales hallazgos empíricos se resumen en los modelos de regresión de mínimos cuadrados ordinarios que se presentan en la tabla 1, en los que la variable dependiente es la carga reguladora. Las 47 observa- 
ciones corresponden a aquellos países de todo el mundo que eran democracias en el periodo 1981-1995 y que tenían una población superior al millón de habitantes. En los tres modelos se mantienen dos variables de control: la variable dicotómica Common Law y el porcentaje de impuestos sobre el PIB. La tercera variable de control, PIB per capita, desaparece en el segundo modelo porque su alta correlación bivariada con la variable rule of law $(0,847)$ invalida el primer modelo por multicolinealidad. Sin embargo -y es necesario subrayar este punto- su desaparición en el segundo modelo relativiza el impacto significativo que presenta la variable rule of law: no sabemos si el impacto es producido por ella misma o porque recoge efectos del PIB.

\section{TABLA 1}

Variable dependiente: carga reguladora (entre paréntesis, error típico)

\begin{tabular}{|c|c|c|c|}
\hline & Modelo 1 & Modelo 2 & Modelo 3 \\
\hline \multirow{2}{*}{ Constante } & $0,462^{* *}$ & $0,519^{* *}$ & 0,157 \\
& $(0,164)$ & $(0,157)$ & $(0,112)$ \\
\hline \multirow{2}{*}{ Common Law } & $-0,040$ & $-0,065$ & $-0,051$ \\
& $(0,107)$ & $(0,106)$ & $(0,105)$ \\
\hline \multirow{2}{*}{ \% impuestos/PIB } & 0,001 & 0,001 & 0,001 \\
\hline PIB capita & $(0,006)$ & $(0,006)$ & $(0,005)$ \\
\hline (en miles de dólares) & 0,016 & & $0,019^{* *}$ \\
\hline \multirow{2}{*}{ Rule of Law } & $(0,014)$ & $0,223^{* *}$ & $(0,150)$ \\
\hline \multirow{2}{*}{ Jugadores con veto } & 0,121 & $(0,077)$ & \\
\hline \multirow{2}{*}{ Inter rule*veto } & $(0,118)$ & 0,050 & \\
\hline
\end{tabular}

** Significativo al $5 \%$. $N=47$

Si comenzamos la interpretación por las variables de control, observamos que ni la existencia de Common Law ni el nivel de intervensionismo estatal, medido a través del porcentaje de impuestos sobre el PIB, parecen tener influencia alguna sobre el nivel de carga reguladora observado en las 47 democracias objeto del estudio. No podemos descartar con ello que exista un impacto de factores de tradición jurídica o administrativa así como que la variable carga reguladora sea en realidad una consecuencia del nivel de intervensionismo estatal en la sociedad civil. Sin embargo, el contraste empírico, en ausencia de mejores indicadores sobre el grado de intervensionismo estatal y la cultura administrativa o jurídica de cada país, no apunta en esa dirección.

El hallazgo más destacado es que ni el sistema judicial (rule of law) ni el número de jugadores con veto parecen tener influencia significativa por separado sobre el nivel de carga reguladora. En el primer modelo resulta obvio, pues ninguna de estas dos variables aparece como significativa. Pero, como he apuntado ya, este modelo debe descartarse por un grave problema de multicolinealidad entre la variable rule of law y el PIB per capita. En el segundo modelo, donde las variables rule of law y jugadores con veto aparecen de nuevo separadas (con la supresión del PIB per capita por el problema de colinealidad), sólo la variable rule of law se muestra significativa. Pero, como también se ha indicado ya, no es posible descartar que la variable rule of law recoja en realidad efectos del nivel de PIB, con lo que la significatividad está bajo sospecha. Por tanto, podemos rechazar la influencia de un impacto individual tanto del número de jugadores con veto como de la eficiencia del sistema judicial sobre la carga reguladora de un país. 
Sin embargo, cuando introducimos una interacción entre las dos variables, como sucede en el modelo 3 , dicha interacción aparece como altamente significativa y con signo positivo. Se trata de una combinación entre la inversa del número de jugadores con veto -el número de jugadores con veto sin transformar tiene signo negativo sobre la variable dependiente y rule of law lo tiene positivo, así que era necesario transformar una de las dosy la variable rule of law. El tamaño del coeficiente $(0,264)$ resulta difícil de interpretar, ya que se trata de la interacción entre la inversa de una variable (jugadores con veto) y otra variable estandarizada (rule of law). Lo que sí se puede afirmar es que la combinación entre un sistema de justicia eficiente con un bajo número de jugadores con veto reduce carga reguladora existente en una sociedad. Los resultados empíricos corroboran, por tanto, la hipótesis apuntada en el modelo formal: el número de jugadores con veto sólo incrementa el nivel de carga reguladora cuando actúa conjuntamente con un sistema judicial ineficiente.

\section{Conclusiones}

Al igual que otras aproximaciones al estudio de la regulación dentro de la Economía Política, este trabajo parte del supuesto de que el exceso de procedimientos administrativos -o la carga reguladora- es una elección de los políticos determinada por la relación de control entre legislativo y ejecutivo. A partir de ese supuesto, se desarrolla un sencillo modelo formal en el que se muestra cómo el legislativo, en una situación de un solo jugador con veto, regulará de manera óptima sin introducir un exceso de regulación que limite la discreción de un ejecutivo que está en manos del mismo partido. En cambio, el legislativo optará por un exceso de legislación cuando, enfrentado con un ejecutivo en manos de otro partido (que definimos como situación de dos jugadores con veto), los costes de introducir un exceso de procedimientos administrativos en la ley no sean demasiado elevados y, siempre y cuando, el sistema judicial sea lo suficientemente ineficiente como para que la lotería de la corrupción tenga valor esperado positivo para el ejecutivo. El contraste empírico corrobora los hallazgos fundamentales del modelo.

Este trabajo tiene dos grandes puntos de partida teóricos. En primer lugar, los estudios que, a partir de los juegos de intereses entre políticos, tratan de explicar la existencia de un exceso de procedimientos administrativos en una sociedad. Sería lo que, en palabras de Moe (1984), podríamos llamar Nueva Economía de la Organización y que recoge para la ciencia políticas hallazgos, como los costes de transacción, de lo que en economía ha supuesto la Nueva Economía Institucional. El otro punto de partida son los recientes intentos de autores, como Epstein y O'Halloran o Huber y Shipan, de construir una teoría comparada de la delegación, tomando al legislativo como principal y a la legislación como mecanismo de delegación básico. A continuación, paso a exponer los avances que, en mi opinión, este trabajo supone para ambas aproximaciones.

Con respecto al primer punto de partida, los resultados de este trabajo expanden el alcance de la teoría de Moe (1990) y, en especial, de Moe y Caldwell (1994), tanto a nivel teórico como empírico. En el plano teórico se produce un doble avance. En primer lugar, se sustituye la dicotomía presidencialismo-parlamentarismo por el concepto de jugadores con veto de Tsebelis (1995), pues no parece que sea la naturaleza constitucional del régimen político, sino los juegos entre adversarios políticos los que conducen a un exceso de procedimientos administrativos. No son los regímenes presidencialistas los que llevan a un exceso de procedimientos, sino el hecho de que exista más de un jugador con veto. En segundo lugar, como se deriva del modelo formal expuesto en el apartado 3, no existirá un incremento de los procedimientos administrativos en una situación de varios jugadores con veto si el sistema judicial es eficiente. Existen, por tanto, países con varios jugadores con veto -tales como EEUU u Holanda- en los que, al haber un sistema judicial eficiente, el nivel de carga reguladora es muy bajo. Por el contrario, en otros contextos con varios jugadores con veto (Italia o Venezuela), la ineficiencia del sistema judi- 
cial conduce a que se legisle con una fuerte carga reguladora. A nivel empírico, este estudio supone también un avance respecto al trabajo de Moe y Ccaldwell. En lugar de explicar el nivel de carga reguladora en los dos casos supuestamente polares de presidencialismo y parlamentarismo (EEUU y Reino Unido), en este trabajo se ofrece un estudio cuantitativo de 47 democracias.

Por otro lado, este trabajo también es deudor de algunas teorías de la delegación (Epstein y O’Halloran 1999; Huber y Shipan 2003). A diferencia de éstas, mi estudio no adopta como variable dependiente el mecanismo de delegación en sí mismo (la ley, medida fundamentalmente en función de su longitud), sino las consecuencias que se derivan para la sociedad del uso de leyes más o menos cargadas de procedimientos administrativos. La introducción de esta nueva variable dependiente supone un avance en un doble sentido. En primer lugar, porque, tal y como se subraya crecientemente desde organizaciones internacionales, el hecho de que una regulación sea óptima o excesiva tiene impacto sobre el desarrollo económico de un país. Por tanto, se estudia una variable de mayor relevancia social que la mera extensión de las leyes. En segundo lugar, porque, como ya he señalado anteriormente, la extensión de la ley puede ser un indicador imperfecto del nivel de discreción que el legislativo cede al ejecutivo fuera del contexto americano, dado el solapamiento de leyes sobre una misma materia o la presencia de detalladas leyes de procedimiento administrativo que observamos en otros sistemas políticos.

En resumen, desde una aproximación de la Economía Política, este artículo presenta una explicación sencilla de las variaciones en el nivel de carga reguladora existentes entre países. La argumentación desarrollada aquí no pretende, no obstante, descartar otras hipótesis alternativas sobre las causas de una mayor o menor carga reguladora. Este estudio no aspira a explicar toda la variación en el nivel de carga reguladora, sino solamente aquella parte de la variación que obedece factores político-institucionales. Es posible que una parte sustancial de la variación descanse en otros factores, como causas históricas o culturales, que quedan fuera de este análisis, fundamentalmente por la dificultad de testar empíricamente esas variables. Independientemente de la validez de esas hipotéticas explicaciones alternativas, en este estudio sí se consigue explicar -a través de un modelo teórico parsimonioso y de un posterior análisis empírico- una parte significativa de la variación en el grado de carga reguladora. 


\section{APÉNDICE}

Tabla de países y sus respectivas puntuaciones en carga reguladora.

\begin{tabular}{|c|c|}
\hline Argentina & ,668 \\
\hline Australia & ,962 \\
\hline Austria & 90 \\
\hline Belgium & ,794 \\
\hline Bolivia &, 876 \\
\hline Brazil & ,134 \\
\hline Canada & 86 \\
\hline Colombia & 29 \\
\hline Costa Rica & ,92 \\
\hline Denmark & 1,048 \\
\hline Ecuador & ,37 \\
\hline Finlandia & 1,140 \\
\hline France &, 713 \\
\hline Germany & ,889 \\
\hline Greece & 60 \\
\hline Honduras & 081 \\
\hline Iceland &, 614 \\
\hline India &,- 040 \\
\hline Ireland & 1,157 \\
\hline Israel &, 533 \\
\hline Italy &, 591 \\
\hline Jamaica & ,760 \\
\hline Japan & ,389 \\
\hline Mauritius & 217 \\
\hline Netherland & 1,141 \\
\hline New Zealand & 1,205 \\
\hline Norway & ,932 \\
\hline Papua New G. &,- 129 \\
\hline Peru & ,669 \\
\hline Portugal & ,889 \\
\hline Spain & ,864 \\
\hline Sweden & 853 \\
\hline Switzerland & 878 \\
\hline Thailand & ,192 \\
\hline Trinidad & ,718 \\
\hline Turkey &, 595 \\
\hline United Kingdom & 1,206 \\
\hline United States & 1,135 \\
\hline Uruguay & ,949 \\
\hline Venezuela & ,090 \\
\hline
\end{tabular}

Fuente: Kaufmann et al., 1999 


\section{REFERENCIAS BIBLIOGRÁFICAS}

Bendor, J. (1988), «Review Article: Formal Models of Bureaucracy», British Journal of Political Science 18: 353-395.

Bendor, J., Glazer, A. y Hammond, Th. (2000), «Theories of Delegation», Annual Review of Political Science 4: 235-269.

Epstein, D. y O'Halloran, S. (1999), Delegating Powers: A Transaction Cost Politics Approach to Policymaking Under Separate Powers. Cambridge: Cambridge University Press.

Geddes, B. (1994), Politician's dilemma: building state capacity in Latin America. Berkeley, Calif.: University of California Press.

Huber, J. D. , Wise and Salutary Neglect. Laws and Bureaucratic Autonomy in Modern Democracies (en vías de publicación).

Kaufman, D., Kraay, A. y Zoido-Lobatón, P. (1999), Governance matters. Washington, D.C: World Bank Institute.

Kiewiet, R. y McCubbins, M.D. (1991c), The logic of delegation: congressional parties and the appropriations process D. Roderick. Chicago, Ill.: University of Chicago Press.

La Porta, R. et al. (1999), «The Quality of Government», Journal of Law, Economics and Organization 15, 1: 222-279.

Moe, T. M. (1984), «The New Economics of Organization», American Journal of Political Science 28: 739-777.

Moe, T. M. (1990), «Political Institution: The Neglected Side of the Story», Journal of Law, Economics, and Organization 6: 213-253.

Moe, T. M. y Caldwell, M. (1994), «The Institutional Foundations of Democratic Government: A Comparison of Presidential and Parliamentary Systems», Journal of Institutional and Theoretical Economics 150: 171-195.

Muller, W. y Strom, K., eds. (2000), Coalition Governments in Western Europe. Nueva York: Oxford University Press.

North, D. (1990), Institutions, institutional change and economic performance. Cambridge: Cambridge University Press.

Scholz, J.T. (1984), «Cooperation, Deterrence, and the Ecology of Regulatory Enforcement», Law and Society Review 18: 601-646.

Scholz, J.T. (1991), «Cooperative Regulatory Enforcement and the Politics of Administrative Effectiveness», American Political Science Review 85, 1: 115-136.

Shipan, Ch. R. (2000), «The Legislative Design of Judicial Review: A Formal Analysis», Journal of Theoretical Politics 12: 269-304.

Strom, K. (2000), «Delegation and Accountability in Parliamentary Democracies», European Journal of Political Research 37, 3: 261 89.

Subirats, J. (1989), Análisis de politicas públicas y eficacia de la Administración. Madrid: INAP.

Tsebelis, G. (1995), «Decision Making in Political Systems: jugadores con veto in Presidentialism, Parliamentarism, Multicameralism and Multipartidism», British Journal of Political Science 25: 289-325.

Tsebelis, G. (2002), Jugadores con veto: How Political Institutions Work. Princeton: Princeton University Press y Russell Sage Foundation.

Weingast, B.R. (1984), «The Congressional-Bureaucratic System: A Principal-Agent Perspective (with Applications to the SEC)», Public Choice 44: 147-191.

World Bank (1997), World development report 1997: the state in a changing world. Washington/Nueva York: Oxford University Press.

World Bank (1999), Entering the 21.t Century, World Development Report 1999/2000. Washington/Nueva York: Oxford University Press.

World Bank (2001), World Development Report 2000/2001, Washington/Nueva York: Oxford University Press. 